\title{
Factors Associated With Survey Response in Hand Surgery Research
}

\author{
Arjan G. J. Bot MD, Jade A. Anderson BS, \\ Valentin Neuhaus MD, David Ring MD, PhD
}

Received: 15 February 2013/Accepted: 17 June 2013/Published online: 26 June 2013

(C) The Association of Bone and Joint Surgeons (B) 2013

\begin{abstract}
Background A low response rate is believed to decrease the validity of survey studies. Factors associated with nonresponse to surveys are poorly characterized in orthopaedic research.

Questions/purposes This study addressed whether (1) psychologic factors; (2) demographics; (3) illness-related factors; and (4) pain are predictors of a lower likelihood of a patient returning a mailed survey.

Methods One hundred four adult, new or return patients completed questionnaires including the Pain Catastrophizing Scale, Patient Health Questionnaire-9 depression scale, Short Health Anxiety Index, demographics, and a pain
\end{abstract}

One of the authors (DR) received a study-specific grant from Skeletal Dynamics (Miami, FL, USA). One of the authors (DR) certifies that he has or may receive payments or benefits, during the study period, an amount of USD 10,000-100,000; an amount less than USD 10,000 from Wright Medical (Arlington, TN, USA), an amount less than USD 10,000 from Skeletal Dynamics (Miami, FL, USA), and an amount less than USD 10,000 from Biomet (Warsaw, IN, USA). One of the authors (AGJB) received a Dutch orthopaedic travel grant,

Anna Fonds Travel grant (Oestgeest, the Netherlands); a nonmedical study grant, VSBfonds (Utrecht, the Netherlands); and a nonmedical study grant for excellent Dutch students, Prins Bernhard

Cultuurfonds/ Banning-de Jong fonds (Amsterdam, the Netherlands). One of the authors (VN) received a grant from Gottfried und Julia Bangerter-Rhyner-Stiftung (Bern, Switzerland).

All ICMJE Conflict of Interest Forms for authors and Clinical Orthopaedics and Related Research editors and board members are on file with the publication and can be viewed on request.

Each author certifies that his or her institution approved the human protocol for this investigation, that all investigations were conducted in conformity with ethical principles of research, and that informed consent for participation in the study was obtained.

This work was performed at the Orthopaedic Hand and Upper Extremity Service, Massachusetts General Hospital, Boston, MA, USA. scale (0-10) during a routine visit to a hand and upper extremity surgeon. Of these patients, $38 \%$ had undergone surgery and the remainder was seen for various other conditions. Six months after their visit, patients were mailed the DASH questionnaire and a scale to rate their satisfaction with the visit (0-10). Bivariate analysis and logistic regression were used to determine risk factors for being a nonresponder to the followup of this study. The cohort consisted of 57 women and 47 men with a mean age of 51 years with various diagnoses. Thirty-five patients (34\%) returned the questionnaire. Responders were satisfied with their visit (mean satisfaction, 8.7) and had a DASH score of 9.6.

A. G. J. Bot, V. Neuhaus, D. Ring ( $\square)$

Harvard Medical School, Orthopaedic Hand and Upper

Extremity Service, Massachusetts General Hospital, Yawkey

Center, Suite 2100, 55 Fruit Street, Boston, MA 02114, USA

e-mail: dring@partners.org

\section{J. A. Anderson}

Boston University School of Medicine, Orthopaedic Hand and Upper Extremity Service, Massachusetts General Hospital, Boston, MA, USA 
Results Compared with patients who returned the questionnaires, nonresponders had higher pain catastrophizing scores, were younger, more frequently male, and had more pain at enrollment. In logistic regression, male sex (odds ratio [OR], 2.6), pain (OR, 1.3), and younger age (OR, 1.03) were associated with not returning the questionnaire. Conclusions Survey studies should be interpreted in light of the fact that patients who do not return questionnaires in a hand surgery practice differ from patients who do return them. Hand surgery studies that rely on questionnaire evaluation remote from study enrollment should include tactics to improve the response of younger, male patients with more pain.

Level of Evidence Level II, prognostic study. See Guidelines for Authors for a complete description of levels of evidence.

\section{Introduction}

One weakness of research that relies on mailed surveys for followup is a low response rate [26]. Response rates after mailings in medical research average approximately $60 \%$ [1]. In our setting (academic hand and upper extremity surgery service in an urban center in the northeastern United States), approximately $1 / 3$ of the patients return surveys $[12,19,20]$. This may limit the external validity of the survey findings $[4,30]$. However, if the response rate is low, it does not imply that the data are not accurate; prior studies showed that a response rate of $20 \%$ in some settings may yield accurate and reliable data $[6,14,32]$.

Previous studies suggest that nonresponders to mailed surveys might be different than responders with respect to age, smoking habit, working status, marital status, and sex $[7,8,13,21]$.

This study addressed whether (1) psychologic factors; (2) demographics; (3) illness-related factors; and (4) pain are predictors of a lower likelihood of a patient returning a mailed survey.

\section{Patients and Methods}

One hundred eight consecutive new or return patients were enrolled in this study, which was approved by our institutional review board. Two patients withdrew, leaving a cohort of 106 patients. Patients were enrolled between April and June 2011. At enrollment, patients completed demographics and the Pain Catastrophizing Scale (PCS), the Patient Health Questionnaire-9 depression scale (PHQ9), the Short Health Anxiety Index (SHAI) [16, 24, 28], and pain questionnaires. They also completed an ordinal health scale in which a number between 0 and 10 was chosen to represent their overall health. Six months after their clinic visit, we sent all patients the DASH questionnaire [11] and a scale to evaluate their satisfaction with their visit (0-10). A prestamped return envelope also was included. The addresses of two patients were incorrect, therefore the final cohort consisted of 104 patients.

The cohort consisted of 57 women (55\%) and 47 men $(45 \%)$ with a mean age of $51 \pm 17$ years at enrollment (range, 18-91 years). The mean duration of pain was $18 \pm 40$ months (range, 0-240 months), and the health scale was $8.4 \pm 1.4$ (range, 4-10). Diagnoses consisted of fractures, carpal tunnel syndrome, osteoarthritis, trigger finger, nonspecific arm pain, and other diagnoses (Table 1). Of the overall cohort, 38\% (39 of 104) were visiting for followup of an earlier surgical procedure, at a mean of 11 months (range, 0.25-96 months) after that procedure.

The PHQ-9 was used to assess depressive symptoms [16]. This questionnaire contains nine questions, answered on a four-point Likert scale; answers range from 0 "not at all" to 3 "nearly everyday" and scores from 0 to 27 are possible [16]. A higher score indicates more symptoms of depression [16]. There were four patients with a total of five missing questions; therefore, we imputed the mean of the patients' other questions for these missing questions. The 13-item PCS was used to measure misinterpretation of nociception (catastrophic thinking) [28]. A higher score indicates a more amplified negative orientation toward pain [28]. There was one missing question for one patient; therefore, we imputed the mean of the patient's answers of the other questions for this missing question.

The SHAI was used to assess health anxiety [24]. This questionnaire has 18 items with scores ranging from 0 to 3 , indicating that a higher total score equaled more health anxiety [24]. The complete SHAI was missing for one patient and we imputed the group mean for this value. There were three patients with a total of six missing questions. We imputed the mean of the patients' other questions for these missing values.

Arm-specific disability at the 6-month mail followup was measured with the DASH [11]. The DASH questionnaire contains 30 questions answered using five-point Likert scales with scores scaled from 0 to 100 points [11]. A higher score indicates greater arm-specific disability. All patients responding to our mailing completed a DASH questionnaire; however, in five patients, this questionnaire was invalid as a result of more than three missing questions [11]. We did not analyze hand dominance. Satisfaction with the visit at our hand clinic was measured with an 11-point ordinal scale. One patient did not complete this evaluation of satisfaction. We did not send reminders to the patients. 
Table 1. Patient demographics $(n=104)$

\begin{tabular}{|c|c|c|c|c|}
\hline Demographic & $\begin{array}{l}\text { Cohort }(\mathrm{n}=104) \\
\text { Mean } \pm \mathrm{SD} \text { (range) }\end{array}$ & $\begin{array}{l}\text { Responders }(\mathrm{n}=35) \\
\text { Mean } \pm \mathrm{SD}(\text { range })\end{array}$ & $\begin{array}{l}\text { Nonresponders }(\mathrm{n}=69) \\
\text { Mean } \pm \mathrm{SD}(\text { range })\end{array}$ & $\mathrm{p}$ value \\
\hline Pain (months) & $18 \pm 40(0-240)$ & $20 \pm 47(0-240)$ & $18 \pm 37(0-240)$ & 0.83 \\
\hline $\begin{array}{l}\text { Age at enrollment } \\
\text { (years) }\end{array}$ & $51 \pm 17(18-91)$ & $56 \pm 17(21-91)$ & $48 \pm 16(18-81)$ & 0.014 \\
\hline Education & $15 \pm 2.6(5-20)$ & $15 \pm 2.3(10-19)$ & $15 \pm 2.8(5-20)$ & 0.82 \\
\hline Health scale & $8.4 \pm 1.4(4-10)$ & $8.7 \pm 1.2(5-10)$ & $8.2 \pm 1.5(4-10)$ & 0.089 \\
\hline \multicolumn{5}{|l|}{ Diagnosis (frequency) } \\
\hline Fracture & $40(39 \%)$ & $14(40 \%)$ & $26(38 \%)$ & \\
\hline $\begin{array}{l}\text { Carpal tunnel } \\
\text { syndrome }\end{array}$ & $12(12 \%)$ & $4(11 \%)$ & $8(12 \%)$ & \\
\hline Osteoarthritis & $9(8.7 \%)$ & $1(2.9 \%)$ & $8(12 \%)$ & \\
\hline Trigger finger & $5(4.8 \%)$ & $2(5.7 \%)$ & $3(4.3 \%)$ & \\
\hline $\begin{array}{l}\text { Nonspecific } \\
\text { arm pain }\end{array}$ & $4(3.8 \%)$ & $2(5.7 \%)$ & $2(2.9 \%)$ & \\
\hline Other & $34(33 \%)$ & $12(34 \%)$ & $22(32 \%)$ & 0.75 \\
\hline \multicolumn{5}{|l|}{ Type of visit } \\
\hline Followup & $64(62 \%)$ & $23(66 \%)$ & $41(59 \%)$ & \\
\hline First visit & $40(39 \%)$ & $12(34 \%)$ & $28(41 \%)$ & 0.53 \\
\hline \multicolumn{5}{|l|}{ Prior surgery } \\
\hline No & $65(63 \%)$ & $19(54 \%)$ & $46(67 \%)$ & \\
\hline Yes & $39(38 \%)$ & $16(46 \%)$ & $23(33 \%)$ & 0.22 \\
\hline \multicolumn{5}{|l|}{ Other pain } \\
\hline No & $67(64 \%)$ & $20(57 \%)$ & $47(68 \%)$ & \\
\hline Yes & $37(36 \%)$ & $15(43 \%)$ & $22(32 \%)$ & 0.27 \\
\hline \multicolumn{5}{|l|}{ Sex } \\
\hline Female & $57(55 \%)$ & $25(71 \%)$ & $32(46 \%)$ & \\
\hline Male & $47(45 \%)$ & $10(29 \%)$ & $37(54 \%)$ & 0.015 \\
\hline \multicolumn{5}{|l|}{ Marital status } \\
\hline Single & $30(29 \%)$ & $7(20 \%)$ & $23(33 \%)$ & \\
\hline Living with partner & $1(1.0 \%)$ & $0(0 \%)$ & $1(1.4 \%)$ & \\
\hline Married & $51(49 \%)$ & $20(57 \%)$ & $31(45 \%)$ & \\
\hline Separated/divorced & $16(15 \%)$ & $6(17 \%)$ & $10(15 \%)$ & \\
\hline Widowed & $6(5.8 \%)$ & $2(5.7 \%)$ & $4(5.8 \%)$ & 0.60 \\
\hline \multicolumn{5}{|l|}{ Work } \\
\hline Full time & $52(50 \%)$ & $15(43 \%)$ & $37(54 \%)$ & \\
\hline Part time & $12(12 \%)$ & $4(11 \%)$ & $8(12 \%)$ & \\
\hline Homemaker & $5(4.8 \%)$ & $1(2.9 \%)$ & $4(5.8 \%)$ & \\
\hline Retired & $21(20.2 \%)$ & $12(34 \%)$ & $9(13 \%)$ & \\
\hline Unemployed & $13(12.5 \%)$ & $3(8.6 \%)$ & $10(15 \%)$ & \\
\hline $\begin{array}{l}\text { Workers } \\
\text { compensation }\end{array}$ & $1(1.0 \%)$ & $0(0 \%)$ & $1(1.4 \%)$ & 0.20 \\
\hline \multicolumn{5}{|l|}{ Smoke } \\
\hline No & $95(91 \%)$ & $34(97 \%)$ & $61(88 \%)$ & \\
\hline Yes & $9(8.7 \%)$ & $1(2.9 \%)$ & $8(11 \%)$ & 0.27 \\
\hline
\end{tabular}

Pain was scored on an 11-point ordinal scale from 0 to 10 in which 0 was no pain and 10 was the worst pain ever experienced. The pain scales for two patients were missing; and we imputed the group mean for these missing values.
An a priori power analysis showed that to detect a medium effect size $(0.6)$ with $80 \%$ power to detect a difference in symptoms of depression between those that returned and did not return the questionnaire with a two- 
tailed independent samples t-test with alpha 0.05 would require 90 patients.

Differences in continuous outcomes (pain in months, age, education, health scale, PHQ-9, PCS, SHAI-18, and pain score) between the responders and nonresponders were assessed with independent samples t-tests.

We assessed differences between categorical variables (diagnosis, type of visit, prior surgery, other pain, sex, marital status, smoking status, and working status) with chi-square tests (and Fisher's exact test when indicated).

All factors with a p less than 0.08 were inserted in a backward stepwise (likelihood) binary logistic regression analysis to find factors associated with returning the mailing. The cut-off value was decided a priori per our routine, which is based on recommendations from our statistician.

\section{Results}

Among the 104 patients, 35 patients (34\%) returned the mailed survey (responders), and 69 patients $(66 \%)$ did not return the survey (nonresponders). The satisfaction with the visit a mean of 7.4 months before was 8.7 of 10 . The mean DASH was 9.6 (range, 0-63) in the 29 patients with a valid DASH (Table 2).

Patients with higher levels of catastrophic thinking (PCS score of $5.6 \pm 7.2$ for nonresponders versus $3.2 \pm 4.9$ points for responders; $\mathrm{p}<0.048$ ), men, younger patients, and patients with greater pain intensity (3.7 versus 2.7; $\mathrm{p}=0.021)$ were less likely to return surveys. There were no differences in health anxiety and depression (Table 3). PCS, sex, age, and pain were entered into a logistic regression of factors associated with not returning the survey. The best model included male sex $(\beta=0.97$, $\mathrm{p}=0.044$; odds ratio $[\mathrm{OR}], 2.6$; CI, 1.03-6.7), younger age $(\beta=0.32, \mathrm{p}=0.031$; OR, 1.03; CI, 1.006-1.060) and greater pain $(\beta=0.26, \mathrm{p}=0.013$; OR, 1.3; CI, 1.06-1.6) (model fit, Hosmer and Lemeshow test: chi-square $=9.6$, $\mathrm{p}=0.29$, Nagelkerke $\mathrm{R}^{2}=0.21$ ) (Table 4). Other factors,

Table 2. Results of responders $(n=35)$

\begin{tabular}{llc}
\hline Variables & $\begin{array}{l}\text { Number of } \\
\text { responses }\end{array}$ & Mean \pm SD (range) \\
\hline Satisfaction & 34 & $8.7 \pm 2.3(0-10)$ \\
Followup (months) & 35 & $7.4 \pm 0.95(5.7-8.9)$ \\
DASH & 29 & $9.6 \pm 14(0-63)$ \\
Subsequent treatment & & \\
$\quad$ No (29 [83\%]) & & \\
Yes $(6[17 \%])$ & & \\
\hline
\end{tabular}

including psychologic factors (depression, health anxiety, catastrophic thinking), demographic factors (work status, education, self-rated health, marital status, smoking), and illness characteristics (diagnosis, time of symptoms, earlier surgery, type of visit) were not associated with a lower likelihood of survey response.

\section{Discussion}

One weakness of studies that use mailed surveys is the frequently low response rate. [1, 15, 17, 21, 25-27]. Previous studies suggest that nonresponders to mailed surveys might differ in important ways from responders [7, 8, 13, 21]. Our study addressed whether (1) psychologic factors; (2) demographic; (3) illness; and (4) pain are predictors of not returning a mailed survey in patients visiting an orthopaedic hand surgery practice. Thirty-four percent of the patients responded to our survey. We found that male sex (OR, 2.6), younger age (OR, 1.03), and greater pain intensity $(\mathrm{OR}, 1.3)$ were associated with no response to a postal survey in this specific study setting.

This study should be viewed in the light of several shortcomings. The cohort was relatively small although sufficiently powered. We had no information regarding why the patients did not return the questionnaire, but that was not the aim of our study. We enrolled patients with various diagnoses, which makes the data more applicable to hand surgery patients in general and allows us to study differences by diagnosis, but might be underpowered for statistical tests of diagnosis and is less well suited to studying the response rate for a population of patients with the same diagnosis. A study with one or a few discrete diagnoses might have different findings, but we think that is unlikely given that diagnosis was not a significant predictor of mailing the survey, although we might not have had adequate power for evaluating diagnosis. The DASH questionnaire was collected only once because we were not studying disability - it was used as a representative questionnaire that we would be likely to ask patients to return by mail. The results of this study might not match those using different questionnaires, shorter followup times, or a different topic.

This study was a prospective cohort study with a relatively short interval between the visit and the mailed survey, which might be expected to have a higher response rate compared with unexpected surveys received by patients years after treatment. However, the percentage loss to followup in our study was higher in comparison to numerous other studies in orthopaedic patients using mailed surveys (range, 56\%-73\% response rate) [3-5, 26, 29, 33].

Most likely the nature of the previous treatment and the specific regional culture are important. For instance, 
Table 3. Descriptives $(n=104)$

\begin{tabular}{|c|c|c|c|c|}
\hline Scale & $\begin{array}{l}\text { Cohort } \\
\text { Mean } \pm \text { SD (range) }\end{array}$ & $\begin{array}{l}\text { Responders }(\mathrm{n}=35) \\
\text { Mean } \pm \mathrm{SD}(\text { range })\end{array}$ & $\begin{array}{l}\text { Nonresponders }(\mathrm{n}=69) \\
\text { Mean } \pm \text { SD }(\text { range })\end{array}$ & $\mathrm{p}$ value \\
\hline Pain & $3.4 \pm 2.4(0-9)$ & $2.7 \pm 1.9(0-8)$ & $3.7 \pm 2.4(0-9)$ & 0.021 \\
\hline PHQ & $3.6 \pm 4.3(0-22)$ & $3.5 \pm 4.4(0-21)$ & $3.8 \pm 4.3(0-22)$ & 0.79 \\
\hline PCS & $4.8 \pm 6.6(0-32)$ & $3.2 \pm 4.9(0-19)$ & $5.6 \pm 7.2(0-32)$ & 0.048 \\
\hline SHAI & $10 \pm 6.4(1-33)$ & $9.8 \pm 5.1(1-21)$ & $10.2 \pm 7.1(1-33)$ & 0.75 \\
\hline
\end{tabular}

PHQ = Patient Health Questionnaire; PCS = Pain Catastrophizing Scale; SHAI = Short Health Anxiety Index.

Table 4. Logistic regression of factors associated with being a nonresponder $(\mathrm{n}=104)$

\begin{tabular}{llllll}
\hline Models & $\beta$ & $\mathrm{p}$ value & $\begin{array}{l}\text { Odds } \\
\text { ratio }\end{array}$ & & \multicolumn{2}{c}{$95 \%$ CI for odds ratio } \\
\cline { 5 - 6 } & & & Lower & Upper \\
\hline \multicolumn{2}{l}{ Model with pain* } \\
Male sex & 0.97 & 0.044 & 2.6 & 1.03 & 6.7 \\
Pain & 0.26 & 0.013 & 1.3 & 1.06 & 1.6 \\
Age & -0.032 & 0.031 & 0.97 & 0.94 & 1.0 \\
& $\beta_{0}=1.1$ & & & &
\end{tabular}

* Variables included in the regression: sex, pain, PCS questionnaire (Pain Catastrophizing Scale), age.

someone who has had complex treatment and has a strong emotional attachment to the care team is probably more likely to respond to the survey than a patient who only met the doctor once and did not have surgery. Satisfaction also might be important [22]. For instance, in a cohort of patients who had a knee arthroplasty, the patients who responded earliest to a mailed survey, containing 10 questions on satisfaction and clinical outcomes, had the highest satisfaction ratings [15]. When the nonresponders were contacted, they were significantly less satisfied compared with the responders [15]. The responding patients in our study were relatively satisfied (8.7 of 10) with their visit so it is possible that the less satisfied patients did not respond to the survey. This is an important unresolved question that requires a different study design to address. Related to this, many survey studies in orthopaedic surgery evaluate the effects of major surgical interventions $[2,5,10,15,17,23,26,27,33]$; ours evaluated a mixed cohort of patients, many of whom had minor interventions or no intervention apart from the office visit. It is difficult to estimate what influence this difference might introduce on our results.

The response rate of $34 \%$ in this prospective cohort was consistent with those of previous retrospective studies $[19,20]$. The factors associated with not returning the survey were younger age, male sex, and more pain. Our finding that older patients are more likely to return the survey is in line with previous studies [7, 21, 26]. Smoking, marital status, and working status were not associated with returning surveys in our study, in contrast to earlier studies that did find differences in these demographic factors [8, 13, 21]. Hardie et al. [9] reported that men were more likely to return postal surveys in a population older than 70 years in contrast to our finding that men were more likely to be nonresponders. There were differences between our study and the study of Hardie et al. [9] which could explain the contrasting outcomes; they evaluated symptoms of respiratory health in a cohort of patients older than 70 years, in a European country and sent the patients a reminder after initial nonresponse.

Solberg et al. sent their patients a survey containing the European Quality Of Life-5 Dimensions (EQ-5D), a question regarding perceived benefit of the operation, and VAS for leg pain, back pain, and state of health after spine surgery [26]. The patients who did not respond were considered to be nonresponders and they received a phone call to find out why they did not return the survey [26]. Reasons for not returning the questionnaire were: they forgot to return the questionnaire or forgot they received a mailing, they were sick of questionnaires, they were ill, or they had family or work problems [26]. However, the outcomes of responders and nonresponders were not significantly different in that study [26]. The outcomes of a study that relies on mailed surveys might be underestimated or overestimated as a result of nonresponders $[15,18,31]$.

We found that patients who did not respond to a single followup mailing were more often male, younger, and had more initial pain. Survey studies should be interpreted in light of the fact that patients who do not return questionnaires in a hand surgery practice differ from patients who do return them. Hand surgery studies that rely on questionnaire evaluation remote from study enrollment should include tactics to improve the response of younger, male patients with more pain.

\section{References}

1. Asch DA, Jedrziewski MK, Christakis NA. Response rates to mail surveys published in medical journals. J Clin Epidemiol. 1997;50:1129-1136.

2. Baker PN, Khaw FM, Kirk LM, Esler CN, Gregg PJ. A randomised controlled trial of cemented versus cementless press-fit 
condylar total knee replacement: 15-year survival analysis. J Bone Joint Surg Br. 2007;89:1608-1614.

3. Bilbao A, Quintana JM, Escobar A, Las Hayas C, Orive M. Validation of a proposed WOMAC short form for patients with hip osteoarthritis. Health Qual Life Outcomes. 2011;9:75.

4. Brown AP, Lawrie HE, Kennedy AD, Webb JA, Torgerson DJ, Grant AM. Cost effectiveness of a prize draw on response to a postal questionnaire: results of a randomised trial among orthopaedic outpatients in Edinburgh. J Epidemiol Community Health. 1997;51:463-464.

5. Cartwright-Terry M, Snow M, Nalwad H. The severity and prediction of anterior knee pain post tibial nail insertion. J Orthop Trauma. 2007;21:381-385.

6. Curtin R, Presser S, Singer E. The effects of response rate changes on the index of consumer sentiment. Public Opin $Q$. 2000;64:413-428.

7. Dallosso HM, Matthews RJ, McGrother CW, Clarke M, Perry SI, Shaw C, Jagger C; Leicestershire MRC Incontinence Study Team. An investigation into nonresponse bias in a postal survey on urinary symptoms. BJU Int. 2003;91:631-636.

8. Gustafsson M, Hagberg L, Holmefur M. Ten years follow-up of health and disability in people with acute traumatic hand injury: pain and cold sensitivity are long-standing problems. $J$ Hand Surg Eur Vol. 2011;36:590-598.

9. Hardie JA, Bakke PS, Morkve O. Non-response bias in a postal questionnaire survey on respiratory health in the old and very old. Scand J Public Health. 2003;31:411-417.

10. Hellum C, Johnsen LG, Storheim K, Nygaard OP, Brox JI, Rossvoll I, Ro M, Sandvik L, Grundnes O; Norwegian Spine Study Group. Surgery with disc prosthesis versus rehabilitation in patients with low back pain and degenerative disc: two year follow-up of randomised study. BMJ. 2011;342:d2786.

11. Hudak PL, Amadio PC, Bombardier C. Development of an upper extremity outcome measure: the DASH (disabilities of the arm, shoulder and hand) [corrected]. The Upper Extremity Collaborative Group (UECG). Am J Ind Med. 1996;29:602-608.

12. Hwang RW, Ring D. Pain and disability related to osteoarthrosis of the trapeziometacarpal joint. J Hand Microsurg. 2011;3:63-65.

13. Jacobsen BK, Thelle DS. The Tromso Heart Study: responders and non-responders to a health questionnaire, do they differ? Scand J Soc Med. 1988;16:101-104.

14. Keeter S, Kennedy C, Dimock M, Best J, Craighill P. Gauging the impact of growing nonresponse on estimates from a national RDD telephone survey. Public Opin Q. 2006;70:759-779.

15. Kim J, Lonner JH, Nelson CL, Lotke PA. Response bias: effect on outcomes evaluation by mail surveys after total knee arthroplasty. J Bone Joint Surg Am. 2004;86:15-21.

16. Kroenke K, Spitzer RL, Williams JB. The PHQ-9: validity of a brief depression severity measure. J Gen Intern Med. 2001;16: 606-613.

17. Kwon SK, Kang YG, Chang CB, Sung SC, Kim TK. Interpretations of the clinical outcomes of the nonresponders to mail surveys in patients after total knee arthroplasty. $J$ Arthroplasty. 2010;25:133-137.
18. Kypri K, Samaranayaka A, Connor J, Langley JD, Maclennan B. Non-response bias in a web-based health behaviour survey of New Zealand tertiary students. Prev Med. 2011;53:274-277.

19. Lozano Calderon SA, Paiva A, Ring D. Patient satisfaction after open carpal tunnel release correlates with depression. J Hand Surg Am. 2008;33:303-307.

20. Lozano-Calderon SA, Souer JS, Jupiter JB, Ring D. Psychological differences between patients that elect operative or nonoperative treatment for trapeziometacarpal joint arthrosis. Hand. 2008;3:271-275.

21. Macera CA, Jackson KL, Davis DR, Kronenfeld JJ, Blair SN. Patterns of non-response to a mail survey. J Clin Epidemiol. 1990;43:1427-1430.

22. McGrath RE, Mitchell M, Kim BH, Hough L. Evidence for response bias as a source of error variance in applied assessment. Psychol Bull. 2010;136:450-470.

23. Rowden NJ, Harrison JA, Graves SE, Miller LN, de Steiger RN, Davidson DC. Loss to follow-up after arthroplasty: a new use for registry data. J Bone Joint Surg Br. 2012;94:493-496.

24. Salkovskis PM, Rimes KA, Warwick HM, Clark DM. The Health Anxiety Inventory: development and validation of scales for the measurement of health anxiety and hypochondriasis. Psychol Med. 2002;32:843-853.

25. Schweitzer M, Asch DA. Timing payments to subjects of mail surveys: cost-effectiveness and bias. J Clin Epidemiol. 1995;48:1325-1329.

26. Solberg TK, Sorlie A, Sjaavik K, Nygaard OP, Ingebrigtsen T. Would loss to follow-up bias the outcome evaluation of patients operated for degenerative disorders of the lumbar spine? Acta Orthop. 2011;82:56-63.

27. Sprague S, Leece P, Bhandari M, Tornetta P 3rd, Schemitsch E, Swiontkowski MF, S.P.R.I.N.T. Investigators. Limiting loss to follow-up in a multicenter randomized trial in orthopedic surgery. Control Clin Trials. 2003;24:719-725.

28. Sullivan MJ, Bishop SR, Scott R, Pivik J. The Pain Catastrophizing Scale: development and validation. Psychol Assess. 1995; 7:525-532.

29. Thwaites J, Mann F, Gilchrist N, McKie J, Sainsbury R. Older patients with hip fractures: evaluation of a long-term specialist orthopaedic medicine service in their outcomes. $N \mathrm{Z} \mathrm{Med} \mathrm{J.}$ 2007; 120:U2535.

30. Ultee J, van Neck JW, Jaquet JB, Hovius SE. Difficulties in conducting a prospective outcome study. Hand Clin. 2003;19:457-462.

31. Verlato G, Melotti R, Olivieri M, Corsico A, Bugiani M, Accordini S, Villani S, Migliore E, Marinoni A, Pirina P, Carrozzi L, Bortolami O, Rava M, de Marco R; ISAYA study group. Asthmatics and exsmokers respond early, heavy smokers respond late to mailed surveys in Italy. Respir Med. 2010;104:172-179.

32. Visser PS, Krosnick JA, Marquette J, Curtin M. Mail surveys for election forecasting? An evaluation of the Colombus Dispatch poll. Public Opin Q. 1996;60:181-227.

33. Wylde V, Blom AW, Whitehouse SL, Taylor AH, Pattison GT, Bannister GC. Patient-reported outcomes after total hip and knee arthroplasty: comparison of midterm results. J Arthroplasty. 2009;24:210-216. 